\title{
Computing Self-Intersections of Closed Geodesics on Finite-Sheeted Covers of the Modular Surface
}

\author{
By J. Lehner and M. Sheingorn*
}

\begin{abstract}
An algorithm is given for deciding whether a closed geodesic on a finite-sheeted cover of the modular surface has self-intersections; if it does, the algorithm gives them in the order they occur in traversing the geodesic. The following general result on geodesics is proved: any closed geodesic on a Riemann surface $R$ can be lifted to a simple closed geodesic on some finite-sheeted cover of $R$. In the last two sections the connection with the stabilizer (under the modular group) of a Markov quadratic irrationality is discussed.
\end{abstract}

0. Introduction. The main object of this paper is to give an algorithm for deciding whether a closed geodesic on a finite-sheeted cover of the modular surface has self-intersections, and if it does, to give them in the order they occur in traversing the geodesic. Birman and Series [3], [4] have a very different approach to this problem, which has yielded nice insights for many Riemann surfaces.

Let $\Gamma(1)=\operatorname{PSL}(2, \mathbf{Z})$ be the modular group and $S_{1}=H^{+} / \Gamma(1)$ the modular surface, where $\mathrm{H}^{+}$is the upper half-plane. If $\Gamma \subset \Gamma(1)$ is of finite index, then $S=H^{+} / \Gamma$ is a finite-sheeted cover of $S_{1}$, and conversely. Let $\sigma \in \Gamma(1)$ be a primitive hyperbolic transformation and $A_{\sigma}$ its axis. $A_{\sigma} / \Gamma(1)$ is a closed geodesic on $S_{1}$; conversely, every such geodesic lifts to a conjugacy class of axes of primitive hyperbolics in $\Gamma(1)$. But because $\Gamma$ is of finite index, $\sigma^{k}$ is in $\Gamma$ for some $k$. Hence $A_{\sigma}$ projects to a closed geodesic on $S$ as well, and this geodesic covers $A_{\sigma} / \Gamma(1)$ on $S_{1}$. Every closed geodesic arises in this way.

Theorem 2.1, which led to our algorithm, provides a relationship between these projections:

THEOREM 2.1. Let $L$ be a closed geodesic on a Riemann surface $R$. Then there is a finite-sheeted cover $S$ of $R$ with the property that $L$ lifts to a simple closed geodesic on $S$.

It should be noted that there are no nontrivial simple closed geodesics on $S_{1}$, so the theorem is never vacuous in that case. For the surface $S_{1}$ and with $S$ a principal congruence subgroup, we had an explicit proof of this result. Morris Newman [7] provided a key algebraic result that made the generalization possible. The proof is in Section 2.

We arrived at the algorithm while searching for simple closed geodesics (scg) on the surface $H^{+} / \Gamma(3)$. This is a sphere with 4 punctures and there are infinitely many

Received March 16, 1984.

1980 Mathematics Subject Classification. Primary 10D05.

* Second author partially supported by NSF and PSC-CUNY grants.

(C)1985 American Mathematical Society $0025-5718 / 85 \$ 1.00+\$ .25$ per page 
homotopy classes, each containing a unique scg. The index of $\Gamma(3)$ in $\Gamma(1)$ is 12 , so the fundamental region (FR) for $\Gamma(3)$ consists of 12 copies of the FR for $\Gamma(1)$. But in the algorithm all the geometry takes place in the FR of $\Gamma(1)$, with its 3 sides at great remove from the real axis. All "irrational" calculation pertains only to this FR. All other calculations involve only integer arithmetic. We believe this is a distinct advantage-one that can be exploited whenever we have a specific subgroup of finite index. The algorithm appears in Section 1.

In our work on $\Gamma(3)$ we are given the endpoints of a hyperbolic axis, i.e., conjugate quadratic irrationals $\alpha, \bar{\alpha}$, but what we need is the primitive hyperbolic transformation of $\Gamma(1)$ fixing $\alpha$ and $\bar{\alpha}$. This calculation, involving the smallest positive solution of a certain Pell equation, is probably well-known, but we could not find it in the literature. It is the subject of Section 3.

To describe the last section, we must define the Markov spectrum (MS) and Markov quadratic irrationalities (MQI). Hurwitz proved that for every irrational $\alpha$ the inequality

$$
\left|\alpha-\frac{p}{q}\right|<\frac{1}{\sqrt{5} q^{2}}
$$

is satisfied by infinitely many reduced fractions and that $\mu_{1}=\sqrt{5}$ is best possible. The value $\sqrt{5}$ is attained for $\alpha \sim \lambda_{1}=(1+\sqrt{5}) / 2$, the equivalence being under the extended modular group (determinant \pm 1 ). For $\alpha+\lambda_{1}$ we can get (0.1) with $\mu_{1}$ replaced by $\mu_{2}=\sqrt{8}$ and this is attained for $\alpha \sim \lambda_{2}=1+\sqrt{2}$. The numbers $\mu_{i}$ increase monotonically to 3 ; they constitute the MS.

The $\lambda_{i}$ are the MQI. They can be described as follows. Consider the Diophantine equation

$$
x^{2}+y^{2}+z^{2}=3 x y z, \quad 1 \leqslant x \leqslant y \leqslant z .
$$

Ordering its solutions by the size of $z$, we have

$$
\mu_{i}=\sqrt{9-\frac{4}{z_{i}^{2}}}, \quad \lambda_{i}=\frac{1}{2}+\frac{y_{i}}{x_{i} z_{i}}+\frac{1}{2} \sqrt{9-\frac{4}{z_{i}^{2}}} .
$$

(Here we assume the well-known conjecture that $z_{i}$ determines $x_{i}$ and $y_{i}$ uniquely.) See Koksma [6, Chapter 3] for all of this.

Halting the algorithm for a given hyperbolic $\sigma \in \Gamma(1)$ depends on finding the least positive $k_{\sigma}=k$ for which $\sigma^{k} \in \Gamma$. When $\Gamma=\Gamma(3)$ we have $\Gamma(1) / \Gamma(3) \simeq A_{4}$, the alternating group on 4 letters. Since $A_{4}$ has no elements of order 4,6 , or 12, we have $k=1,2$, or 3 [8, p. 31]. Our computations, however, suggested that for $\sigma$ fixing $\lambda_{i}$, an MQI, $k=2$. In Section 4 we prove that, for such $\sigma, k \leqslant 2$, and that proving $k=2$ depends on a congruence property of the smallest solution of the corresponding Pell equation.

Lastly, we mention that using our algorithm on various $\lambda_{i}$ suggested the conjecture:

$$
\begin{aligned}
& A_{\sigma} \text { projects to a simple closed geodesic on } H^{+} / \Gamma(3) \\
& \text { if and only if } \sigma \text { fixes a MQI. }
\end{aligned}
$$

Using methods of number theory, topology, and differential geometry we were subsequently able to prove this conjecture. (See [2].) Combined with an asymptotic formula for the number of $z_{i} \leqslant X$ given by Zagier [10], (0.4) gives upper and lower bounds for the number of scg on $H^{+} / \Gamma(3)$ whose hyperbolic length is $\leqslant X$. 
1. An Algorithm for Finding Self-Intersections of a Closed Geodesic on a Modular Surface of Finite Index in $\Gamma(1)$. We shall assume that the closed geodesic is given as the axis of an explicit hyperbolic transformation $A$. We can also assume the axis intersects the standard fundamental region (SFR) for $\Gamma(1)$, entering at a point $z_{0}$ that lies on a vertical side of the SFR. This can be achieved by an effective conjugation of $A$. Thus we have fixed points of $A, \xi_{A}<\xi_{A}^{\prime}$, that satisfy $\xi_{A}^{\prime}-\xi_{A}>1$.

Since our surface is of finite index, there is a least positive integer $n_{A}=n$ such that $A^{n} \in \Gamma$. Our implementations of this algorithm test each integer $n=1,2,3, \ldots$ to see if $A^{n} \in \Gamma$. For many subgroups of $\Gamma(1)$ there is a very simple membership test (Rankin [8, pp. 63-65]).

We now have determined $A, n=n_{A}, \xi=\xi_{A}$, and $\xi^{\prime}=\xi_{A}^{\prime}$. We are going to follow the axis from $z_{0}$ towards $A^{n}\left(z_{0}\right)$. As we first pass out of the SFR at $z_{1}$, say, we determine which of $S=(1,1: 0,1), S^{-1}$, or $T=(0,-1: 1,0)$ returns the path (from $z_{1}$ towards $\left.A^{n}\left(z_{0}\right)\right)$ to the SFR. Call this $L_{1}$. Apply $L_{1}$ to the axis of $A$; the image enters the SFR at $L_{1}\left(z_{1}\right)$ and moves towards $L_{1} \circ A^{n}\left(z_{0}\right)$. Again we leave the SFR at $z_{2}$ and map the segment proceeding from $z_{2}$ towards $L_{1} \circ A^{n}\left(z_{0}\right)$ back to the SFR by $L_{2}$, one of $S, S^{-1}$, or $T$. Continuing in this way, we obtain a sequence $\left\{L_{j}\right\}$, $j=1, \ldots, k=k\left(A^{n}\right)$. Furthermore, $A^{n}=L_{k} \circ L_{k-1} \circ \cdots \circ L_{2} \circ L_{1}$, since the segments emanating from $z_{0}$ and $A^{n}\left(z_{0}\right)$ are $\Gamma$-equivalent and a fortiori $\Gamma(1)$-equivalent. Because of the relation $(T S)^{3}=I$, there are many representations of $A^{n}$ as a word in the generators $S$ and $T$, but the word obtained as above is about as short as possible (Beardon [1, Theorem 5]).

Our first task is to determine the $L$ 's. We assume we are proceeding from $z_{0}$ to $\xi$, the other case being no different. Find $m$ such that $m-\frac{1}{2}<\xi<m+\frac{1}{2}$. By our normalization, $m \leqslant 0$. Proceeding from $z_{0}$ towards $\xi$, we have $|m|$ or $|m|-1$ instances of $S$ to begin our sequence of $L$ 's, according as we are in case (a) or (b) in Figure 1:

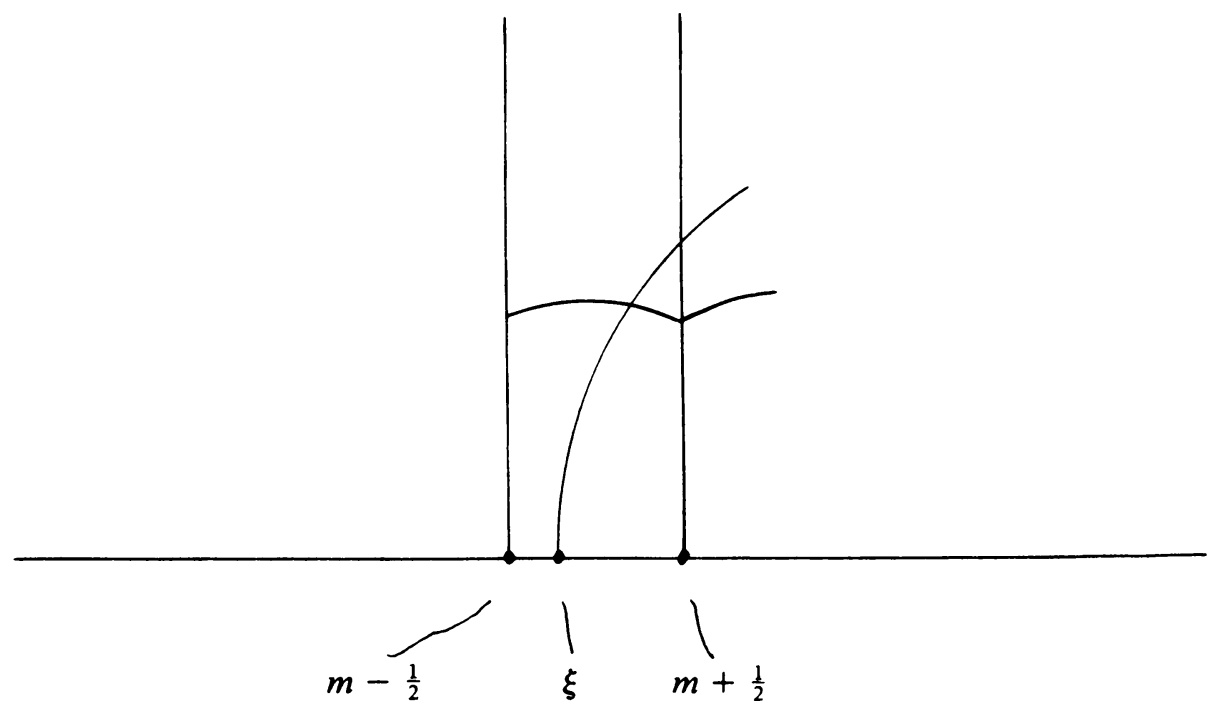

FIGURE 1 (a) 


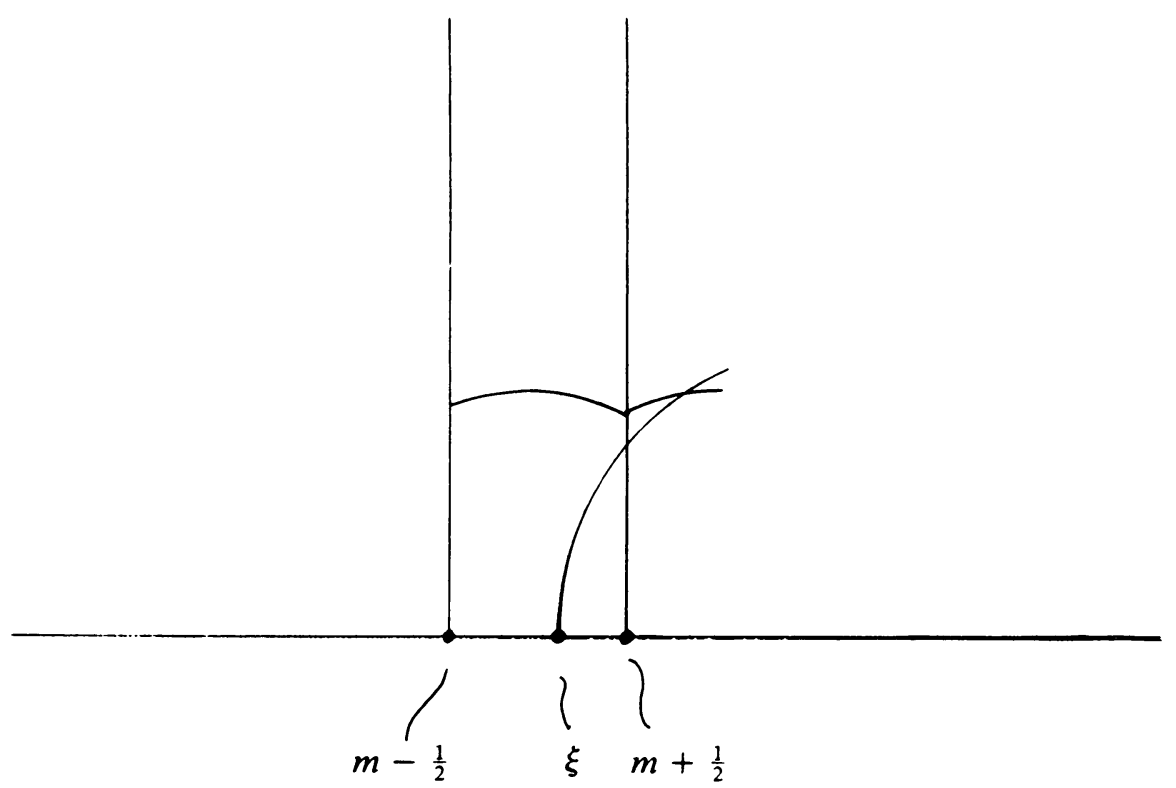

FIGURE 1 (b)

Determining the intersection of the axis with $x=m+\frac{1}{2}$ (solving a quadratic equation) distinguishes the cases. We include the limiting case in which the intersection occurs at height $\sqrt{3} / 2$ in case (b).

After this initial string of $S$ 's, the next $L$ is of course $T$. Calculate $T \circ S^{|m|}(\xi)=\xi_{1}$ and $T \circ S^{|m|}\left(\xi^{\prime}\right)=\xi_{1}^{\prime}$, and determine whether or not $\xi_{1}<\xi_{1}^{\prime}$. We know we are continuing from $z_{1}$ towards $\xi_{1}$. Now just as with our analysis from $z_{0}$, we obtain a sequence of transformations $S$ or $S^{-1}$ according as $\xi_{1}<\xi_{1}^{\prime}$ or not. The number of $L$ 's in this sequence is $\left|m_{1}\right|$ or $\left|m_{1}\right|-1$, where $m_{1}-\frac{1}{2}<\xi_{1}<m_{1}+\frac{1}{2}$, as before. The next $L$ is $T$.

We continue in this way until $\xi_{t}=\xi$ and $\xi_{t}^{\prime}=\xi^{\prime}$ in this order. (Note that if $\xi_{t}=\xi^{\prime}, \xi_{t}^{\prime}=\xi$ occurs, it means that the axis has fixed points of order 2 on it.) Up to this point we have determined $L_{1}, \ldots, L_{t}$ and we know that $A=L_{t} \circ \ldots \circ L_{1}$, since the product fixes $\xi, \xi^{\prime}$ and is primitive because $\xi_{t}=\xi, \xi_{t}^{\prime}=\xi^{\prime}$ does not occur earlier. The full sequence of $L$ 's is just $L_{1}, \ldots, L_{t}$ repeated $n$-times.

Next, define $Q_{k}=L_{k} \circ \cdots \circ L_{1}$. We see that $Q_{k}$ maps a segment of the axis between $z_{0}$ and $A\left(z_{0}\right)$ into the SFR. These $Q_{k}$ are the only elements of $\Gamma(1)$ with this property. This means that if $\beta$ and $\beta^{\prime}$ lie in the segment of the axis between $z_{0}$ and $A\left(z_{0}\right)$ with $\beta$ nearer $z_{0}$, and $\beta$ is $\Gamma(1)$-equivalent to $\beta^{\prime}$, then $Q_{k}(\beta)=Q_{j}\left(\beta^{\prime}\right)$ for some $j>k$. Thus $\beta=Q_{k}^{-1} \circ Q_{j}\left(\beta^{\prime}\right)$.

Now we compute all $Q_{k}^{-1} \circ Q_{j}, n t \geqslant j>k$, and check for $\Gamma(1)$-self-intersections. This involves computing $\xi_{k, j}:=Q_{k}^{-1} \circ Q_{j}(\xi)$ and $\xi_{k, j}^{\prime}:=Q_{k}^{-1} \circ Q_{j}\left(\xi^{\prime}\right)$ and checking for the following 4 incidence patterns (recall $\xi<\xi^{\prime}$ ):
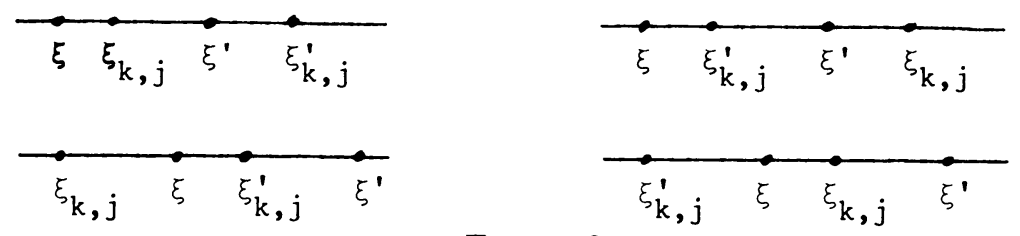

Figure 2 
If a self-intersection occurs, compute it to make sure it lies in $\left[z_{0}, A^{n} z_{0}\right]$-this avoids finding the same intersection more than once. We now have a set of self-intersections ordered by the distance of $\beta$ from $z_{0}$. This sequence actually gives all $\Gamma(1)$-intersections in the order they occur in traversing the axis from $z_{0}$ to $A^{n}\left(z_{0}\right)$, which is $n$-times around the curve $A / \Gamma(1)$.

Finally, to find $\Gamma$-intersections, simply check which of the $\Gamma(1)$-intersections have $Q_{k}^{-1} \circ Q_{j} \in \Gamma$. The efficacy of this algorithm lies in the fact that the geometry is carried out on the SFR of $\Gamma(1)$ with its 2 simple identifications and 4 long sides whose 3 vertices are far from the real axis. $\Gamma$ enters only in that one needs to check membership therein-an integer arithmetic problem for many congruence subgroups. This is much preferable to dealing with a fundamental region with many short sides and closely-spaced vertices near the real axis, as the algorithm of Poincaré requires [9, pp. 192-194].

2. Proof of the Lifting Theorem. As we have said, this theorem relies on a result of M. Newman.

THeOREM 2.2. Let $\Gamma$ be a Fuchsian group, $\left\{\alpha_{1}, \ldots, \alpha_{k}\right\}$ a finite subset of $\Gamma$ not containing $1, \gamma \in \Gamma$ such that $\gamma \alpha_{i} \neq \alpha_{i} \gamma, 1 \leqslant i \leqslant k$. Then there is a subgroup $\Gamma_{1}$ of $\Gamma$ such that $\left(\Gamma: \Gamma_{1}\right)<\infty, \gamma \in \Gamma_{1}, \alpha_{i} \notin \Gamma_{1}, i=1, \ldots, k$.

Proof of Theorem 2.1. Let the Riemann surface $R=H^{+} / \Gamma$, where $\Gamma$ is a Fuchsian group, and let $\pi: H^{+} \rightarrow R$ be the projection map. Let $L$ be a closed geodesic in $R$. We observe that $L$ can have only finitely many self-intersections, since a geodesic on a surface of constant negative curvature is a real analytic curve. Now $L$ lifts to a countable set of axes of primitive hyperbolic elements in $\Gamma$. Let $\gamma$ be one such with axis $A_{\gamma}$.

Choose a point $a$ on $A_{\gamma}$ such that $\pi(a)$ is not a self-intersection of $L$, and let $b=\gamma(a)$. As we move from $a$ to $b$ along $A_{\gamma}$, the projection traverses $L$ once. Also, moving from $a$ to $b$ we pass through finitely many fundamental regions of $\Gamma$. Hence there are at most finitely many $1 \neq \alpha_{i} \in \Gamma$ with $\alpha_{i}(z)=z^{\prime} ; z, z^{\prime} \in[a, b)$. Note that $\alpha_{i} \gamma \neq \gamma \alpha_{i}$, as else $\alpha_{i}$ and $\gamma$ would have the same fixed points, implying that $\alpha_{i}=\gamma^{m}$, $m \neq 0$. This is not possible, as $z, z^{\prime} \in[a, b)$.

Now apply Theorem 2.2, obtaining a finite-index subgroup $\Gamma_{1}$ of $\Gamma$ that contains $\gamma$ but excludes $\left\{\alpha_{i}\right\}$. Clearly $\gamma$ is primitive also in $\Gamma_{1}$. On the surface $S:=H^{+} / \Gamma_{1}$, the axis $A_{\gamma}$ projects to a closed geodesic $L_{1}$ and $L_{1}$ is described once as $A_{\gamma}$ is traversed from $a$ to $b$.

We claim that $A_{\gamma} / \Gamma_{1}$ is simple. If not, there is a $\sigma \in \Gamma_{1}$ with

$$
A_{\gamma} \neq \sigma\left(A_{\gamma}\right) \cap A_{\gamma} \neq \varnothing \text {. }
$$

In particular, $\sigma \neq 1$. Let $\sigma\left(A_{\gamma}\right) \cap A_{\gamma}=z_{1} \in H^{+}$. Without loss of generality, $z_{1}$ $\in[a, b)$.

Let $z_{1}=\sigma\left(z_{2}\right)$ with $z_{2} \in A_{\gamma}$. There exist integers $m, n$ such that $\gamma^{m}\left(z_{1}\right), \gamma^{n}\left(z_{2}\right)=$ $\gamma^{n} \circ \sigma^{-1}\left(z_{1}\right) \in[a, b)$. Since they are $\Gamma_{1}$-equivalent and both lie in $[a, b)$, Theorem 2.2 forces $\gamma^{n} \circ \sigma^{-1}=\gamma^{m}$, or

$$
\gamma^{n-m}=\sigma
$$


Whether $n=m$ or $n \neq m$, this contradicts (2.1). Finally, since $\Gamma_{1} \subset \Gamma, A_{\gamma} / \Gamma_{1}$ covers $A_{\gamma} / \Gamma$.

\section{Constructing a Generator for the $\Gamma(1)$-Stabilizer of a Quadratic Irrationality.}

THEOREM 3.1. Let $\alpha$ be a quadratic irrationality whose primitive polynomial is

$$
a_{2} z^{2}+a_{1} z+a_{0}=0, \quad a_{2} \neq 0, \quad\left(a_{2}, a_{1}, a_{0}\right)=1 .
$$

Then the $\Gamma(1)$-stabilizer of $\alpha$ is generated by

$$
\left(\begin{array}{cc}
\frac{L-K a_{1}}{2} & -K a_{0} \\
K a_{2} & \frac{L+K a_{1}}{2}
\end{array}\right),
$$

where $(L, K)$ is the positive solution of the Pell equation

$$
K^{2}\left(a_{1}^{2}-4 a_{0} a_{2}\right)+4=L^{2}
$$

with minimal $K$ (and thus minimal $L)$.

Remark. In what follows, we are regarding $\Gamma(1)$ as a transformation group, i.e., we identify $V \in \Gamma(1)$ with $-V$.

Proof. Every quadratic polynomial with roots $\alpha, \bar{\alpha}$ is of the form

and conversely.

$$
u a_{2} x^{2}+u a_{1} x+u a_{0}=0, \quad u \neq 0,
$$

Now suppose $V=(a, b: c, d) \in \Gamma(1)$ has fixed points $\alpha, \bar{\alpha}$. Then

$$
c \alpha^{2}+(d-a) \alpha-b=0 \text {. }
$$

Hence,

$$
c=K a_{2}, \quad d-a=K a_{1}, \quad-b=K a_{0}, \quad K \neq 0,
$$

where clearly $K$ is rational. Let $K=K_{1} / K_{2},\left(K_{1}, K_{2}\right)=1$. We have $K_{2} c=K_{1} a_{2}$, yielding $K_{2} \mid a_{2}$. Similarly, $K_{2}\left|a_{1}, K_{2}\right| a_{0}$. Since $\left(a_{2}, a_{1}, a_{0}\right)=1$, we must have $K_{2}=1$, i.e., $K \in \mathbf{Z}$. Hence, every $V=(a, b: c, d) \in \Gamma(1)$ with fixed points $\alpha, \bar{\alpha}$ satisfies (3.3) with an integer $K$. Furthermore, there exists an integer $L$ such that

$$
K^{2}\left(a_{1}^{2}-4 a_{0} a_{2}\right)+4=L^{2} .
$$

The last statement is proved as follows. We have

$$
\begin{gathered}
(a+d)^{2}-(a-d)^{2}=4+4 b c=4-4 K^{2} a_{0} a_{2}, \\
(a+d)^{2}=4+K^{2}\left(a_{1}^{2}-4 a_{0} a_{2}\right)=L^{2}
\end{gathered}
$$

for an integer $L$, since $a+d \in \mathbf{Z}$. Note that $K a_{1}$ and $L$ have the same parity.

Conversely, if $a, b, c, d \in \mathbf{Z}$ satisfy (3.3) and (3.4) for some integers $K, L$, then $V=(a, b: c, d) \in \Gamma(1)$ and has fixed points $\alpha, \bar{\alpha}$. This is easily checked. So we have

LEMMA 3.2. The subgroup $\Gamma_{\alpha}$ consists of the $V \in \Gamma$ satisfying (3.1) and (3.2). Here we have written $\Gamma$ for $\Gamma(1)$. 
An element of $\Gamma_{\alpha}$ is therefore of the form (3.2), where we may assume $K, L>0$. Let $L_{1}$ be the smallest solution of (3.4) and write $K_{1}$ for the corresponding $K$. Let

$$
V_{1}=\left(\begin{array}{cc}
\left(L_{1}-K_{1} a_{1}\right) / 2 & -K_{1} a_{0} \\
K a_{2} & \left(L_{1}+K_{1} a_{1}\right) / 2
\end{array}\right)
$$

We shall show that $V_{1}$ generates $\Gamma_{\alpha}$.

LEMMA 3.3. Let $A=(\alpha, \beta: \gamma, \delta) \in \operatorname{SL}(2, \mathbf{R})$ be hyperbolic. Then,

$$
\begin{gathered}
A^{n}=u_{n} A+v_{n} I, \quad v_{n+1}=-u_{n}, \quad n \geqslant 0 ; \quad u_{0}=0, \quad v_{0}=1, \\
u_{n}=2^{n-1} \sinh n \theta / \sinh \theta, \quad n \geqslant 1, \theta>0
\end{gathered}
$$

where $\theta$ is defined by

$$
\alpha+\delta= \pm 2 \cosh \theta
$$

The proof is by induction on $n$, using $A^{2}=(\alpha+\delta) A-I$.

Proceeding with the proof of the theorem, we let $W$ be a generator of the cyclic group $\Gamma_{\alpha}$, where, as noted above, we regard $\Gamma$ as a transformation group. Then

$$
W=\left(\begin{array}{cc}
\left(L_{0}-K_{0} a_{1}\right) / 2 & -K_{0} a_{0} \\
K_{0} a_{2} & \left(L_{0}+K_{0} a_{1}\right) / 2
\end{array}\right)
$$

for a solution $\left(K_{0}, L_{0}\right)$ of (3.4). Suppose $V_{1}=W^{n}, n \geqslant 1$. Then, by Lemmas 3.2 and 3.3,

$$
\left|K_{1} a_{2}\right|=\left|u_{n} K_{0} a_{2}\right|=2^{n-1} \frac{\sinh n \theta}{\sinh \theta}\left|K_{0} a_{2}\right|, \quad\left|K_{1}\right| \geqslant\left|K_{0}\right|
$$

By hypothesis $\left|K_{1}\right| \leqslant\left|K_{0}\right|$, hence $\left|K_{1}\right|=\left|K_{0}\right|$, which implies $n=1$. That is, $V_{1}=W$, a generator. This concludes the proof of Theorem 3.1.

4. The Exponent of a Markov Quadratic Irrationality. A Markov quadratic irrationality (MQI) is a number of the form

$$
\alpha=\frac{1}{2}+\frac{y}{x z}+\frac{1}{2} \sqrt{9-\frac{4}{z^{2}}},
$$

where $x, y, z(x \leqslant y \leqslant z)$ is a Markov triple. Writing out the equation satisfied by $\alpha$ and cancelling common factors in the coefficients, we find, by comparison with (3.1), that

$$
a_{2}=\frac{x^{2} z}{(z, 2)}, \quad a_{1}=\frac{x(x z+2 y)}{(z, 2)}, \quad a_{0}=\frac{-2 x^{2} z+4 x y-z}{(z, 2)}
$$

Here we have used the coprimality of $x, y, z$ in pairs (Cassels [5, p. 28]). 
Now (4.2) and Theorem 3.1 give

THEOREM 4.1. With $\alpha$ as in (4.1) we have that the primitive matrix $B_{\alpha}$ generating the stabilizer of $\alpha$ in $\Gamma(1)$ is

$$
\left(\begin{array}{cc}
\frac{L+x(2 y+x z) K}{2 d} & K\left(2 x^{2} z-4 x y+z\right) d^{-1} \\
x^{2} z K d^{-1} & \frac{L-x(2 y+x z) K}{2 d}
\end{array}\right)
$$

where $d=(z, 2) . L$ and $K$ are defined as in Theorem 3.1; explicitly $K$ (and therefore $L$ ) is the minimal positive solution of the Pell equation

$$
x^{4}\left(9 z^{2}-4\right) d^{-2} K^{2}+4=L^{2} .
$$

Moreover, the smallest power of $B_{\alpha}$ lying in $\Gamma(3)$ is 1 or 2 according as $(3, K)=3$ or 1 .

Proof. Only the last statement needs proof. We easily deduce from the Markov equation that $3+x z$. Equation (4.4) now forces $3 \mid K L, K \equiv L$ (3). Assume $3 \mid L$, then $L \mid L d^{-1}=$ trace $B_{\alpha}$. Hence,

$$
B_{\alpha}^{2} \equiv\left(\begin{array}{cc}
-1 & 0 \\
0 & -1
\end{array}\right)(\bmod 3)
$$

i.e., $B_{\alpha}^{2} \in \Gamma(3)$. Next, let $3+L$, then $3 \mid K$. It follows that

$$
B_{\alpha} \equiv\left(\begin{array}{ll}
1 & 0 \\
0 & 1
\end{array}\right) \quad \text { or } \quad\left(\begin{array}{cc}
-1 & 0 \\
0 & -1
\end{array}\right)(\bmod 3)
$$

i.e., $B_{\alpha} \in \Gamma(3)$. Finally, $3+K$ implies $B_{\alpha} \notin \Gamma(3)$ since $x^{2} z K d^{-1} \not \equiv 0$ (3). This completes the proof.

School of Mathematics

Institute for Advanced Study

Princeton, New Jersey 08540

Department of Mathematics

Baruch College

City University of New York

New York, New York 10010

1. A. F. BEARDON, "The structure of words in discrete subgroups of $\operatorname{SL}(2, C)$, , J. London Math. Soc. (2), v. 10, 1975, pp. 201-211.

2. A. F. BeARdon, J. Lehner \& M. Sheingorn, "Closed simple geodesics on Riemann surfaces and the Markov spectrum." (To be published.)

3. J. Birman \& C. SERies, “An algorithm for simple curves on surfaces,” J. London Math. Soc. (2), v. 29, 1984, pp. 331-342.

4. J. Birman \& C. Series, "Simple curves have Hausdorff dimension one." (Preprint.)

5. J. W. S. CASsels, An Introduction to Diophantine Approximation, Cambridge Univ. Press, Cambridge, 1957.

6. J. F. Koksma, Diophantische Approximationen, Springer-Verlag, Berlin, 1936; reprinted, Chelsea, New York.

7. M. Newman, “A note on fuchsian groups,” Illinois J. Math. (To appear).

8. R. A. Rankin, Modular Forms, Cambridge Univ. Press, Cambridge, 1977.

9. J. Stillwell, Classical Topology and Combinatorial Group Theory, Graduate Texts in Mathematics, No. 72, Springer-Verlag, Berlin and New York, 1980.

10. D. ZAGIER, "On the number of Markoff numbers below a given bound," Math. Comp., v. 39, 1982, pp. 709-723. 